\title{
On the feasibility of wireless energy transfer using massive antenna arrays in Rician channels
}

Salil Kashyap, Emil Björnson and Erik G Larsson

The self-archived postprint version of this conference article is available at Linköping University Institutional Repository (DiVA):

http:/ / urn.kb.se/ resolve?urn=urn:nbn:se:liu:diva-128669

N.B.: When citing this work, cite the original publication.

Kashyap, S., Björnson, E., Larsson, E. G, (2015), On the feasibility of wireless energy transfer using massive antenna arrays in Rician channels, 16th IEEE International Workshop on Signal Processing Advances in Wireless Communications, SPAWC 2015 , 46-50.

https:// doi.org/ 10.1109/ SPAWC.2015.7226997

Original publication available at:

https:/ / doi.org/ 10.1109/ SPAWC.2015.7226997

Copyright: Institute of Electrical and Electronics Engineers (IEEE)

http:/ / www.ieee.org/

(C)2015 IEEE. Personal use of this material is permitted. However, permission to reprint/republish this material for advertising or promotional purposes or for creating new collective works for resale or redistribution to servers or lists, or to reuse any copyrighted component of this work in other works must be obtained from the IEEE. 


\title{
On the Feasibility of Wireless Energy Transfer Using Massive Antenna Arrays in Rician Channels
}

\author{
Salil Kashyap, Emil Björnson, and Erik G. Larsson \\ Dept. of Electrical Eng. (ISY), Linköping University, 58183 Linköping, Sweden \\ Email: \{salil.kashyap, emil.bjornson, erik.g.larsson\}@liu.se
}

\begin{abstract}
In this paper, we examine the feasibility of wireless energy transfer (WET) using arrays with multiple antennas. Specifically, we compute the probability of outage in energy transfer over a Rician fading channel when the base station (BS) with multiple antennas transfers energy to a wireless sensor node (WSN). Through our analytical and numerical results, we prove that by deploying more antennas at the BS, the range of WET can be increased while maintaining a target outage probability. We observe that the use of massive antenna arrays at the BS results into huge savings of radiated energy. We show that for typical energy levels used in WET, the outage performance with imperfect channel state information (CSI) is essentially the same as that obtained based on perfect CSI. We also observe that a strong line-of-sight component between the BS and the WSN lowers the probability of outage in energy transfer.
\end{abstract}

Index Terms-Wireless energy transfer, massive MIMO, beamforming, outage probability, array gain

\section{INTRODUCTION}

Wireless energy transfer (WET) is a promising energy harvesting technology where the destination node harvests energy from electromagnetic radiations [1], [2]. However, there are several challenges that must be addressed in order to implement WET. Firstly, only a small fraction of the energy radiated by an energy transmitter can be harvested by the wireless sensor node (WSN) which severely limits the range of WET [3], [4]. Secondly, the received energy levels that are suitable for wireless information transfer are often not suitable for WET, where the absolute received energy is of interest and not the signal-to-noise ratio. To significantly boost the performance of WET, large transmitter arrays can be used [1], [5]. Massive multiple input multiple output (MIMO) systems, where the base station (BS) employs antenna arrays with a few hundred antennas, have recently emerged as a leading $5 \mathrm{G}$ wireless communications technology. They provide orders of magnitude higher data rates and energy efficiency than current wireless systems [6].

Contributions: We consider a network where a BS equipped with a massive antenna array communicates with and beamforms (radio-frequency) RF energy to a WSN. The motivation behind using an antenna array is that it can make use of the array gain, resulting from coherent combination of the signals transmitted from each antenna, provided it knows its channel to the WSN. This array gain in turn may lead to an extension of the operating range and/or reduction in the amount of transmit energy needed to satisfy a given energy harvesting constraint. The drawback is that the wireless

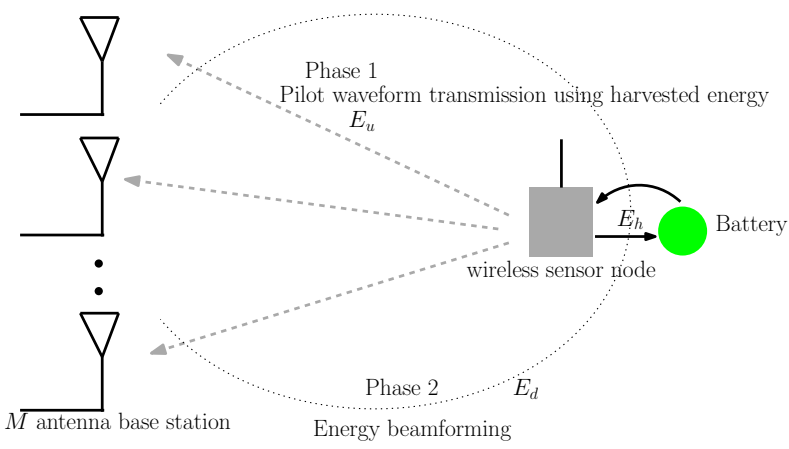

Fig. 1. The two phase communication protocol.

channel between the BS and the WSN fluctuates so that the channel state information (CSI) needs to be acquired on a regular basis to enable coherent combining.

As shown in Figure 1, the communication between the BS and the WSN takes place in two phases. In the first phase, the WSN transmits a known pilot waveform using the energy stored in a battery or a capacitor. This is measured at each antenna in the BS array, in order to estimate the CSI from the sensor. In the second phase, the BS performs transmit beamforming of energy to the WSN, using the estimated CSI and exploiting channel reciprocity. The energy harvested by the WSN is used to recharge its capacitor or battery, and needed in turn for pilot transmission in phase one of the next round and also to perform the main tasks of the sensor. Moreover, both phases may involve transfer of information, although that is not the focus of this paper.

The main objective of this paper is to estimate the link budget in order to ascertain the viability of a system that performs WET using massive antenna arrays. We investigate the following specific questions: 1) what array gain can the massive MIMO setup provide in WET, i.e., how does the required radiated energy scale with the number of antennas in the array given that the CSI is estimated from pilots? 2) how does the number of antennas at the BS depend on the path loss or the distance between the BS and the WSN? 3) how do the answers to the previous questions depend on propagation conditions? To this end, we compute new, exact expressions for the probability of outage in energy transfer, defined here as the probability that the energy harvested by the WSN is less than the energy that it spends on uplink pilot signaling, for both perfect and imperfect CSI and for a general Rician fading channel model. 
Related Work: In order to maximize the net average harvested energy at the WSN, while [7] derived the optimal uplink pilot power and the number of antennas at the WSN that need to be trained, [8] looked at optimizing the time that must be allocated for pilot signaling and energy transfer. In [9], a wireless powered communication network with one multiantenna BS and a set of single antenna users was considered for joint downlink energy transfer and uplink information transmission via space division multiple access. Simultaneous wireless information and power transfer for a multiuser multiple input single output (MISO) system, where a multi-antenna BS sends information and energy simultaneously to several single antenna users which then perform information decoding or energy harvesting was studied in [1], [5], [10]. However, none of these papers consider the possibility of an outage in energy transfer. To address outages in energy transfer, [11] analyzed the probability of outage in energy transfer for a MISO system over a Rayleigh fading channel and with CSI acquired using least squares channel estimation. In this paper, we extend the analysis of [11] to a more general Rician fading channel model and with minimum mean square error (MMSE) channel estimation.

\section{SySTEM MODEL}

We consider a frequency-flat, block-fading channel model in which the channel impulse response from each antenna at the BS to the WSN remains constant during a coherence interval of length $\tau$ seconds. The channel realizations are randomly generated and they are independent across blocks. We, therefore, need to estimate it after every coherence interval. We assume time-division duplexing (TDD) mode of communication so that the channel from the BS to the WSN referred to as the downlink channel is the same as the channel from the WSN to the BS referred to as the uplink channel. Therefore, the BS can take advantage of channel reciprocity and make channel measurements using uplink signals.

We focus on a wireless network where a BS with $M$ antennas is used to transfer RF energy to a single antenna WSN that has energy harvesting capabilities. We consider a scenario where a line-of-sight (LoS) link might be present between the BS and the WSN and for which the channel $h$ from the BS to the WSN can be modeled by the Rician fading model as [12]

$$
\boldsymbol{h}=\sqrt{\frac{K}{K+1}} h_{d}+\sqrt{\frac{1}{K+1}} h_{s},
$$

where $\boldsymbol{h}_{d} \in \mathbb{C}^{M \times 1}$ is the deterministic vector containing the specular components of the channel, $K$ is the Rician factor defined as the ratio of the deterministic to the scattered power, and $\boldsymbol{h}_{s} \in \mathbb{C}^{M \times 1}$ denotes the scattered components of the channel and is a random vector with i.i.d. zero mean unit variance circular symmetric complex Gaussian entries. Furthermore, $\boldsymbol{h}_{d}=\left[\begin{array}{llll}1 & e^{j \theta_{1}(\phi)} & \cdots & e^{j \theta_{(M-1)}(\phi)}\end{array}\right]^{T}$ where $\theta_{i}(\phi), i=$ $1, \ldots, M-1$ is the phase shift of the $i^{\text {th }}$ antenna with respect to the reference antenna and $\phi$ is the angle of departure/arrival of the specular component. Thus, $\boldsymbol{h} \sim \mathcal{C N}\left(\boldsymbol{\mu}, \Lambda_{h}\right)$, where $\boldsymbol{\mu}=$ $\sqrt{\frac{K}{K+1}}\left[\begin{array}{llll}1 & e^{j \theta_{1}(\phi)} & \cdots & e^{j \theta_{(M-1)}(\phi)}\end{array}\right]^{T}$ and $\boldsymbol{\Lambda}_{h}=\frac{1}{K+1} \mathbf{I}_{M}$.

\section{A. Uplink Pilot Signaling and Channel Estimation}

The signal ${ }^{1} \boldsymbol{y}(t)$ that is received at the BS when the WSN transmits a continuous-time pilot signal $\sqrt{E_{u}} p(t)$ of duration $T$ such that $\int_{0}^{T}|p(t)|^{2} d t=1$, is given by

$$
\boldsymbol{y}(t)=\sqrt{\beta} \sqrt{E_{u}} \boldsymbol{h} p(t)+\boldsymbol{w}(t), \text { for } t \in[0, T],
$$

where $\beta$ denotes the distance-dependent path loss, $E_{u}$ is the energy spent on uplink pilot signaling in Joule, $\boldsymbol{h} \in \mathbb{C}^{M \times 1}$ is the channel as defined in (1) and $\boldsymbol{w}(t)$ is the thermal noise vector at the BS that is independent of $\boldsymbol{h}$. The objective of the uplink pilot signaling is to estimate $\boldsymbol{h}$ given $\boldsymbol{y}(t)$.

Now, a sufficient statistic for estimating $\boldsymbol{h}$ at the BS is $\boldsymbol{y}=\int_{0}^{T} p^{*}(t) \boldsymbol{y}(t) d t=\sqrt{\beta} \sqrt{E_{u}} \boldsymbol{h}+\boldsymbol{w}$, where $\boldsymbol{w} \in \mathbb{C}^{M \mathrm{x} 1}$ is circular symmetric complex additive white Gaussian noise (AWGN). Furthermore, $\boldsymbol{w} \sim \mathcal{C N}\left(\mathbf{0}, N_{0} \mathbf{I}_{M}\right)$, where $N_{0}$ is the noise power spectral density in Joule. Thus, given $y$, the MMSE estimate $\widehat{\boldsymbol{h}}_{\text {MMSE }}$ of $\boldsymbol{h}$ is given by [13]

$$
\widehat{\boldsymbol{h}}_{\mathrm{MMSE}}=\mathbb{E}_{\boldsymbol{h}}[\boldsymbol{h} \mid \boldsymbol{y}]=\mathbb{E}_{\boldsymbol{h}}[\boldsymbol{h}]+\boldsymbol{\Sigma}_{\boldsymbol{h y}} \boldsymbol{\Sigma}_{\boldsymbol{y}}^{-1}\left(\boldsymbol{y}-\mathbb{E}_{\boldsymbol{y}}[\boldsymbol{y}]\right),
$$

where $\boldsymbol{\Sigma}_{\boldsymbol{h} \boldsymbol{y}}$ is the cross-covariance matrix of $\boldsymbol{h}$ and $\boldsymbol{y}$ and $\boldsymbol{\Sigma}_{\boldsymbol{y}}$ is the covariance matrix of $\boldsymbol{y}$. It is straightforward to show that $\boldsymbol{\Sigma}_{\boldsymbol{h y}}=\frac{\sqrt{\beta} \sqrt{E_{u}}}{K+1} \mathbf{I}_{M}$ and $\boldsymbol{\Sigma}_{\boldsymbol{y}}=\frac{\beta E_{u}+(K+1) N_{0}}{K+1} \mathbf{I}_{M}$. Using these, (3) can be simplified to obtain

$$
\widehat{\boldsymbol{h}}_{\mathrm{MMSE}}=\boldsymbol{h}+\widetilde{\boldsymbol{h}}_{\mathrm{MMSE}}
$$

where $\widetilde{\boldsymbol{h}}_{\mathrm{MMSE}} \sim \mathcal{C N}\left(\mathbf{0}, \frac{N_{0}}{\beta E_{u}+(K+1) N_{0}} \mathbf{I}_{M}\right)$ is the estimation error that is independent of $\widehat{\boldsymbol{h}}_{\mathrm{MMSE}}$.

\section{B. Transmit Beamforming Based on the MMSE Estimate}

Given the MMSE channel estimate $\widehat{\boldsymbol{h}}_{\text {MMSE }}$, the BS performs transmit beamforming of energy, i.e., it aligns the signals emitted from the different antennas so that they add up coherently at the WSN in order to maximize the harvested received energy. Therefore, on the downlink, it transmits $\boldsymbol{x}(t)=\sqrt{E_{d}} \frac{\widehat{\boldsymbol{h}}_{\mathrm{MMSE}}^{\dagger}}{\left\|\widehat{\boldsymbol{h}}_{\mathrm{MMSE}}\right\|} p^{\prime}(t)$ where $E_{d}$ is the downlink energy in Joule, $(\cdot)^{\dagger}$ is the conjugate transpose, and $p^{\prime}(t)$ is a unit energy continuous-time pulse of duration $T^{\prime}$.

The WSN receives a continuous-time signal $y^{\prime}(t)$ on the downlink and it is given by

$$
y^{\prime}(t)=\sqrt{\beta} \sqrt{E_{d}} \frac{\widehat{\boldsymbol{h}}_{\mathrm{MMSE}}^{\dagger} \boldsymbol{h}}{\left\|\widehat{\boldsymbol{h}}_{\mathrm{MMSE}}\right\|} p^{\prime}(t)+w^{\prime}(t), \text { for } t \in\left[0, T^{\prime}\right],
$$

where $w^{\prime}(t)$ is the thermal noise at the WSN. We denote the energy harvesting efficiency of the WSN by $\eta$. Then, the

\footnotetext{
${ }^{1}$ This is the complex baseband representation of a physical quantity that is proportional to the voltage measured across the load connected to the BS antenna. The proportionality constant in turn depends on the load resistor used.
} 
energy harvested $E_{h}$ in Joule based on the MMSE channel estimate is

$$
E_{h}=\eta \beta E_{d}\left|\frac{\widehat{\boldsymbol{h}}_{\mathrm{MMSE}}^{\dagger} \boldsymbol{h}}{\left\|\widehat{\boldsymbol{h}}_{\mathrm{MMSE}}\right\|}\right|^{2}
$$

Note that $E_{h}$ is a random variable (RV). Moreover, the contribution from $w^{\prime}(t)$ to $E_{h}$ is neglected, since it cannot be harvested. Let

$$
\Psi_{\mathrm{MMSE}} \triangleq \frac{\widehat{\boldsymbol{h}}_{\mathrm{MMSE}}^{\dagger} \boldsymbol{h}}{\left\|\widehat{\boldsymbol{h}}_{\mathrm{MMSE}}\right\|} .
$$

We state below the conditional statistics of $\Psi_{\text {MMSE }}$ that will be used in the analysis in Section III-B.

Lemma 1: Given the MMSE channel estimate $\widehat{\boldsymbol{h}}_{\mathrm{MMSE}}$, $\Psi_{\text {MMSE }}$ is a complex Gaussian RV with conditional mean

$$
\mathbb{E}\left[\Psi_{\mathrm{MMSE}} \mid \widehat{\boldsymbol{h}}_{\mathrm{MMSE}}\right]=\left\|\widehat{\boldsymbol{h}}_{\mathrm{MMSE}}\right\|,
$$

and conditional variance

$$
\operatorname{var}\left[\Psi_{\text {MMSE }} \mid \widehat{\boldsymbol{h}}_{\mathrm{MMSE}}\right]=\frac{N_{0}}{\beta E_{u}+(K+1) N_{0}} .
$$

Proof: This follows from standard results on conditional Gaussian RVs and we omit the proof due to space constraints.

\section{Probability of OUtage in Energy Transfer}

In this section, we compute the probability of outage in energy transfer first for the scenario when the BS has perfect CSI about its link to the WSN and then for imperfect CSI based on MMSE channel estimation. We define the "probability of outage in energy transfer" $P_{O}$ as the probability that the energy harvested $E_{h}$ by the WSN is less than or equal to the energy $E_{u}$ that it spends on uplink pilot signaling, i.e.,

$$
P_{o}=\operatorname{Pr}\left(E_{h} \leq E_{u}\right) \text {. }
$$

As a baseline, we also consider the case of perfect CSI, in which case the BS knows $\boldsymbol{h}$ completely. This reference case gives us a bound in terms of the best outage performance that can be achieved and we include it to understand when the uplink pilots are the limiting factor. In the analysis below, we assume that $E_{d}$ is fixed and not adapted based on the channel conditions.

\section{A. Analysis with Perfect CSI}

As mentioned before, the channel estimation is considered error-free if we spend $E_{u}$ on uplink pilot signaling and there is no noise in the estimation process. The probability of outage in energy transfer in such a scenario is as follows:

Theorem 1: The probability of outage in energy transfer with perfect CSI is

$$
P_{o}=1-Q_{M}\left(\sqrt{2 K M}, \sqrt{\frac{2(K+1) E_{u}}{\eta \beta E_{d}}}\right),
$$

where $Q_{M}(\cdot, \cdot)$ is the $M^{\text {th }}$ order Marcum-Q function [14, Eqn. (4.59)].
Proof: The proof is given in Appendix A.

Note that the outage probability for a given $M, \eta, K$ and $\beta$ in (11) does not change as long as the ratio of $E_{u}$ to $E_{d}$ remains the same.

\section{B. Analysis with MMSE Channel Estimation}

With MMSE channel estimation, the probability of outage in energy transfer is given by the following result.

Theorem 2: For a Rician fading channel $(K \neq 0)$, the probability of outage in energy transfer with MMSE channel estimation is

$$
\begin{gathered}
P_{o}=1-\frac{2 \Lambda(K+1)^{\frac{M+1}{2}}}{(K M)^{\frac{M-1}{2}}} \exp (-\Lambda K M) \\
\times \int_{0}^{\infty} y^{M} \exp \left(-\Lambda(K+1) y^{2}\right) I_{M-1}(2 \Lambda \sqrt{K(K+1) M} y) \\
\times Q_{1}\left(\sqrt{\Lambda_{0}} y, \sqrt{\frac{\Lambda_{0} E_{u}}{\eta \beta E_{d}}}\right) d y . \quad(12)
\end{gathered}
$$

where $I_{M-1}(\cdot, \cdot)$ is the $(M-1)^{\text {th }}$ order modified Bessel function of first kind [14, Eqn. (4.36)], $Q_{1}(\cdot, \cdot)$ is the first order Marcum-Q function [14, Eqn. (4.33)], $\Lambda=\frac{\beta E_{u}+(K+1) N_{0}}{\beta E_{u}}$, and $\Lambda_{0}=\frac{2\left(\beta E_{u}+(K+1) N_{0}\right)}{N_{0}}$.

Proof: The proof is given in Appendix B.

Note that (12) is in the form of a single integral in $y$ and probably cannot be simplified any further as the integrand involves the product of a modified Bessel function and a Marcum-Q function. It is, however, easy to evaluate numerically. The case where $K=0$ was considered in [11].

Based on Theorems 1 and 2,

- For a fixed $M, K, E_{u}, \eta$, and $\beta$, using (11) for perfect CSI, or (12) for MMSE estimation, one can compute the energy $E_{d}$ with which the downlink energy-bearing signals must be transmitted, so that a target probability of outage in energy transfer is satisfied.

- One can conclude how the required value of $M$ scales with the path loss $\beta$ or with the distance between the BS and the WSN, for a given $P_{o}$.

- One can quantify the loss due to channel estimation errors.

- One can evaluate the role played by the LoS component, i.e., the Rician- $K$ factor on the outage probability.

\section{NumERICAL RESULTS}

In this section, we present numerical results to quantify the potential of using massive antenna arrays for WET using the two phase communication scheme in Figure 1. Unless mentioned otherwise, we take $E_{u}=10^{-8} \mathrm{~J}(100 \mu \mathrm{W}$ during $100 \mu \mathrm{s}), E_{d}=10^{-3} \mathrm{~J}(1 \mathrm{~W}$ during $1 \mathrm{~ms}), \eta=0.5$ and $N_{0}=k_{B} T 10^{F / 10}=10^{-20} \mathrm{~J}$, where $k_{B}=1.38 \times 10^{-23} \mathrm{~J} / \mathrm{K}$, $T=300 \mathrm{~K}$, and the receiver noise figure $F=7 \mathrm{~dB}$. We vary $\beta$ around a nominal value of $-50 \mathrm{~dB}$, which according to experimental results reported in [4] corresponds to a 2.2 meter BSWSN separation in an office corridor environment. Also, we consider a uniform linear array for which $\theta_{i}(\phi)=2 \pi d i \cos \phi$, 


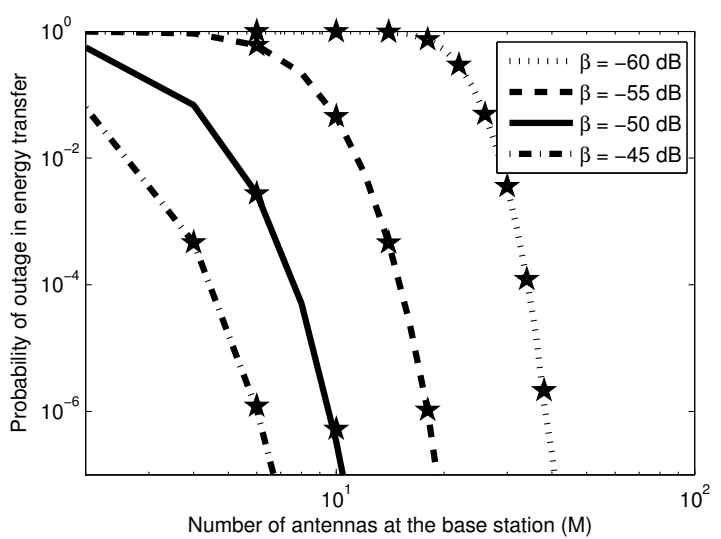

Fig. 2. Impact of $\beta$ and $M$ on $P_{o}\left(E_{d}=10^{-3} \mathrm{~J}, E_{u}=10^{-8} \mathrm{~J}, N_{0}=\right.$ $10^{-20} \mathrm{~J}, K=2$, and $\left.\eta=0.5\right)$. The corresponding perfect CSI results are shown using ' $\star$ '.

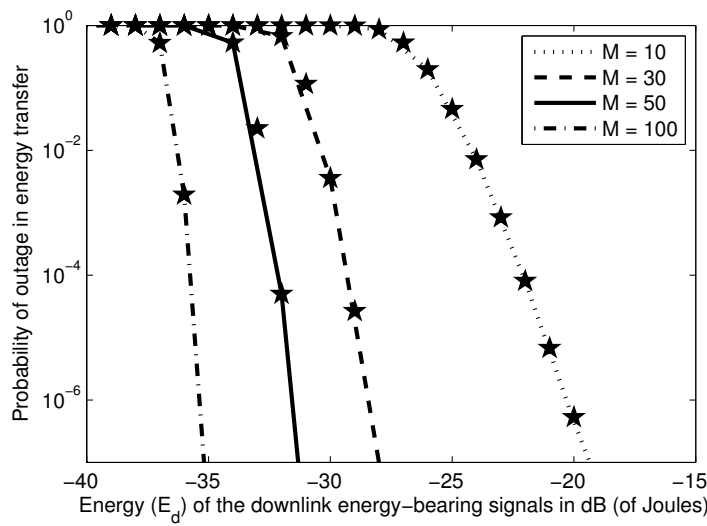

Fig. 3. Impact of $E_{d}$ and $M$ on $P_{o}\left(\beta=-60 \mathrm{~dB}, E_{u}=10^{-8} \mathrm{~J}, N_{0}=\right.$ $10^{-20} \mathrm{~J}, K=2$, and $\left.\eta=0.5\right)$. The corresponding perfect CSI results are shown using ' $\star$ '.

$i=1, \ldots, M-1$. We take $\phi=\pi / 3$ and $d=\frac{\lambda}{2}=0.06 \mathrm{~m}$, where $\lambda$ is the wavelength at a frequency of $2.45 \mathrm{GHz}$.

We plot the analytical result for the probability of outage in energy transfer for the MMSE estimator obtained using (12) and for the perfect CSI obtained using (11) in the figures below. We have cross-checked our analytical expressions against the corresponding Monte Carlo simulation results and they are in perfect agreement with each other. We do not show them here just to avoid clutter.

Figure 2 plots $P_{o}$ as a function of $M$ for different values of the path loss $\beta$ and for $K=2$. We observe that by deploying more antennas at the BS, a larger path loss (larger distance between the BS and the WSN) can be tolerated while keeping the outage probability fixed. For example, by going from about 10 antennas to 40 antennas at the BS, an outage probability of $10^{-6}$ can be maintained even if the path loss increases from $50 \mathrm{~dB}$ to $60 \mathrm{~dB}$. Also, for $E_{u}=10^{-8} \mathrm{~J}$ and $E_{d}=10^{-3} \mathrm{~J}$, the performance is basically the same as that obtained from perfect CSI.

Figure 3 plots $P_{o}$ as a function of $E_{d}$ for different $M$ and

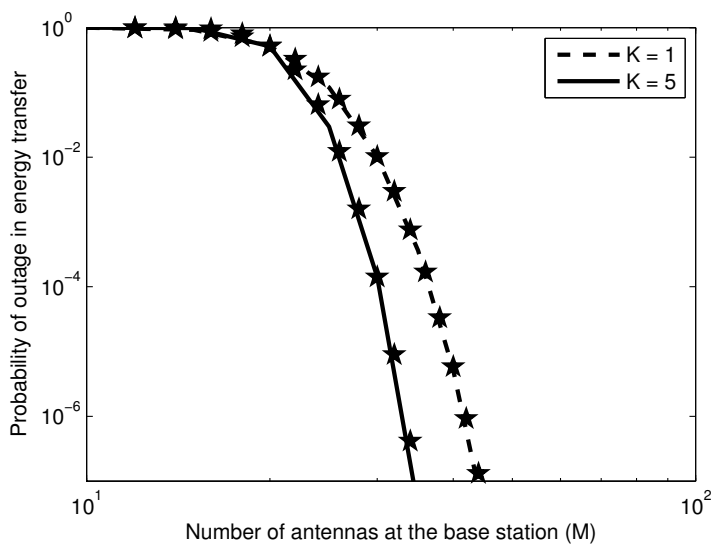

Fig. 4. Impact of $K$ and $M$ on $P_{o}\left(E_{u}=10^{-8} \mathrm{~J}, E_{d}=10^{-3} \mathrm{~J}\right.$, $\beta=-60 \mathrm{~dB}, N_{0}=10^{-20} \mathrm{~J}$, and $\left.\eta=0.5\right)$. The corresponding perfect CSI results are shown using ' $\star$ '.

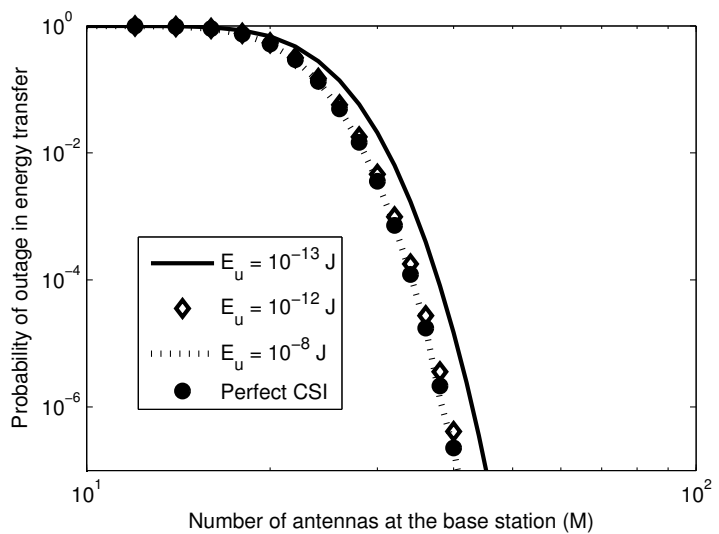

Fig. 5. Impact of $E_{u}$ and $M$ on $P_{o}$ keeping $\frac{E_{u}}{E_{d}}$ fixed at $10^{-5}\left(N_{0}=\right.$ $10^{-20} \mathrm{~J}, \beta=-60 \mathrm{~dB}, K=2$, and $\left.\eta=0.5\right)$.

for $K=2$. It can be observed that as $E_{d}$ increases, the outage probability decreases. Moreover, as more antennas are deployed at the BS, a lower $E_{d}$ is required to keep the outage probability at the same value. For example, by going from about 10 to 30 antennas at the BS, $E_{d}$ can be reduced by $6 \mathrm{~dB}$, while keeping the outage probability fixed at $10^{-6}$. Thus, the array gain obtained by deploying multiple antennas at the BS results in huge savings of radiated energy. One can also see that the outage performance is the same as with perfect CSI.

Figure 4 plots $P_{o}$ as a function of $M$ for two different values of the Rician- $K$ factor, namely, $K=1$ and $K=5$ and for both perfect and imperfect CSI obtained again using MMSE channel estimation. It can be observed that as $K$ increases, the channel becomes more deterministic and the outage probability improves with both perfect and imperfect CSI. In other words, a strong line-of-sight component in the channel helps in lowering the outage probability.

Figure 5 plots $P_{o}$ as a function of $M$ for different values of $E_{u}$, keeping the ratio of $E_{u}$ to $E_{d}$ fixed at $10^{-5}$. We keep the ratio fixed to evaluate the effect of channel estimation errors 
on $P_{o}$ (cf. (11)). It can be observed that for $E_{u}=10^{-8} \mathrm{~J}$ (100 $\mu \mathrm{W}$ for $100 \mu \mathrm{s})$, the outage probability is the same as with perfect CSI, i.e., estimation errors do not degrade the performance. Also, estimation errors lead to a degradation in the outage probability only if $E_{u}$ is $10^{-13} \mathrm{~J}$ or lower. However, such energy levels are very small to enable WET and for the sensor to be able to harvest any reasonable amount of energy.

\section{CONCLUSIONS}

We evaluated the viability of using a massive antenna array for WET using the two phase communication scheme. To this end, we derived new, exact expressions for the outage probability when the BS uses an array with multiple antennas to focus and transfer energy to a WSN. We proved that by deploying more antennas at the BS, the range for WET can be increased while satisfying a given target outage probability constraint. We observed that huge savings of radiated energy can be obtained by deploying more antennas at the BS. We further observed that for typical energy levels that are used in WET, the outage probability with imperfect CSI is the same as that with perfect CSI. Finally, we saw that a strong LoS component between the BS and the WSN helps to improve the outage probability.

\section{APPENDIX}

\section{A. Proof of Theorem 1}

With perfect CSI, $\Psi=\Psi_{\mathrm{MMSE}}=\frac{\widehat{\boldsymbol{h}}_{\mathrm{MMSE}}^{\dagger} \boldsymbol{h}}{\left\|\widehat{\boldsymbol{h}}_{\mathrm{MMSE}}\right\|}=\|\boldsymbol{h}\|$, where $\widehat{\boldsymbol{h}}_{\mathrm{MMSE}}=\boldsymbol{h}$ is the MMSE channel estimate. Thus,

$$
\begin{aligned}
P_{o} & =\operatorname{Pr}\left(\eta \beta E_{d}|\Psi|^{2} \leq E_{u}\right), \\
& =\operatorname{Pr}\left(2(K+1)\|\boldsymbol{h}\|^{2} \leq \frac{2(K+1) E_{u}}{\eta \beta E_{d}}\right) .
\end{aligned}
$$

Using the fact that $2(K+1)\|\boldsymbol{h}\|^{2}$ is a non-central chi-square distributed RV with $2 M$ degrees of freedom and non-centrality parameter $2 K M,(11)$ is obtained from the CDF expression.

\section{B. Proof of Theorem 2}

With MMSE channel estimation,

$$
P_{o}=\mathbb{E}_{\widehat{\boldsymbol{h}}_{\mathrm{MMSE}}}\left[\operatorname{Pr}\left(\left|\Psi_{\mathrm{MMSE}}\right|^{2} \leq \frac{E_{u}}{\eta \beta E_{d}} \mid \widehat{\boldsymbol{h}}_{\mathrm{MMSE}}\right)\right] .
$$

Let

$$
\widetilde{\Psi}_{\mathrm{MMSE}}=\frac{\Psi_{\mathrm{MMSE}}}{\sqrt{\frac{N_{0}}{2\left(\beta E_{u}+(K+1) N_{0}\right)}}} .
$$

Therefore, (15) reduces to $P_{o}$

$=\mathbb{E}_{\widehat{\boldsymbol{h}}_{\text {MMSE }}}\left[\operatorname{Pr}\left(\left|\widetilde{\Psi}_{\mathrm{MMSE}}\right|^{2} \leq \frac{2 E_{u}\left(\beta E_{u}+(K+1) N_{0}\right)}{\eta \beta E_{d} N_{0}} \mid \widehat{\boldsymbol{h}}_{\mathrm{MMSE}}\right)\right]$.

Given $\widehat{\boldsymbol{h}}_{\mathrm{MMSE}}, \operatorname{Re}\left(\widetilde{\Psi}_{\mathrm{MMSE}}\right)$ and $\operatorname{Im}\left(\widetilde{\Psi}_{\mathrm{MMSE}}\right)$ are independent Gaussian RVs. Using Lemma 1 , it can be shown that $\mathbb{E}\left[\operatorname{Re}\left(\widetilde{\Psi}_{\mathrm{MMSE}}\right) \mid \widehat{\boldsymbol{h}}_{\mathrm{MMSE}}\right]=\sqrt{2\left(\frac{\beta E_{u}+(K+1) N_{0}}{N_{0}}\right)}\left\|\widehat{\boldsymbol{h}}_{\mathrm{MMSE}}\right\|$, $\mathbb{E}\left[\operatorname{Im}\left(\widetilde{\Psi}_{\mathrm{MMSE}}\right) \mid \widehat{\boldsymbol{h}}_{\mathrm{MMSE}}\right]=0$, and the conditional variances are given by $\operatorname{var}\left[\operatorname{Re}\left(\widetilde{\Psi}_{\mathrm{MMSE}}\right) \mid \widehat{\boldsymbol{h}}_{\mathrm{MMSE}}\right]=$
$\operatorname{var}\left[\operatorname{Im}\left(\widetilde{\Psi}_{\text {MMSE }}\right) \mid \widehat{\boldsymbol{h}}_{\text {MMSE }}\right]=1$. Thus, given $\widehat{\boldsymbol{h}}_{\text {MMSE }}$, $\left|\widetilde{\Psi}_{\text {MMSE }}\right|^{2}$ is a non-central chi-square distributed RV with 2 degrees of freedom and non-centrality parameter $2\left(\frac{\beta E_{u}+(K+1) N_{0}}{N_{0}}\right)\left\|\widehat{\boldsymbol{h}}_{\mathrm{MMSE}}\right\|^{2}$. Therefore, (17) reduces to

$$
P_{o}=\mathbb{E}_{\widehat{\boldsymbol{h}}_{\mathrm{MMSE}}}\left[1-Q_{1}\left(\sqrt{\Lambda_{0}}\left\|\widehat{\boldsymbol{h}}_{\mathrm{MMSE}}\right\|, \sqrt{\frac{\Lambda_{0} E_{u}}{\eta \beta E_{d}}}\right)\right],
$$

where $\Lambda_{0}=2\left(\frac{\beta E_{u}+(K+1) N_{0}}{N_{0}}\right)$.

To compute (18), we need to find the distribution of $Y=\left\|\widehat{\boldsymbol{h}}_{\mathrm{MMSE}}\right\|=\sqrt{\left|\hat{h}_{\mathrm{MMSE}_{1}}\right|^{2}+\cdots+\left|\hat{h}_{\mathrm{MMSE}_{M}}\right|^{2}}$. It can be shown that $\frac{2(K+1)\left(\beta E_{u}+(K+1) N_{0}\right)}{\beta E_{u}} Y^{2}$ is a non-central chisquare distributed $\mathrm{RV}$ with $2 M$ degrees of freedom and noncentrality parameter $\frac{2 K M\left(\beta E_{u}+(K+1) N_{0}\right)}{\beta E_{u}}$. Also, by transformation of RVs, $Y=\left\|\widehat{\boldsymbol{h}}_{\mathrm{MMSE}}\right\|$ will have the following PDF

$$
\begin{aligned}
& f_{Y}(y)=\frac{2 \Lambda(K+1)^{\frac{M+1}{2}}}{(K M)^{\frac{M-1}{2}}} \exp (-\Lambda K M) y^{M} \\
& \times \exp \left(-\Lambda(K+1) y^{2}\right) I_{M-1}(2 \Lambda \sqrt{K(K+1) M} y),
\end{aligned}
$$

where $\Lambda=\frac{\beta E_{u}+(K+1) N_{0}}{\beta E_{u}}$. Substituting the PDF of $Y$ from (19) in (18) yields (12).

\section{REFERENCES}

[1] I. Krikidis, S. Timotheou, S. Nikolaou, G. Zheng, D. W. K. Ng, and R. Schober, "Simultaneous wireless information and power transfer in modern communication systems,"' IEEE Commun. Mag., vol. 52, no. 11, pp. 104-110, Nov. 2014.

[2] X. Lu, P. Wang, D. Niyato, D. I. Kim, and Z. Han, "Wireless networks with RF energy harvesting: A contemporary survey," Available: http://arxiv.org/abs/1406.6470, 2014.

[3] Z. Popovic, E. A. Falkenstein, D. Costinett, and R. Zane, "Low-power far-field wireless powering for wireless sensors," Proc. IEEE, vol. 101, no. 6, pp. 1397-1409, Jun. 2013.

[4] H. J. Visser and R. J. M. Vullers, "RF energy harvesting and transport for wireless sensor network applications: Principles and requirements,' Proc. IEEE, vol. 101, no. 6, pp. 1410-1423, Jun. 2013.

[5] X. Chen, Z. Zhang, H.-H. Chen, and H. Zhang, "Enhancing wireless information and power transfer by exploiting multi-antenna techniques," Available: http://arxiv.org/abs/1501.02429, 2015.

[6] E. G. Larsson, O. Edfors, F. Tufvesson, and T. Marzetta, "Massive MIMO for next generation wireless systems," IEEE Commun. Mag., vol. 52, no. 2, pp. 186-195, Feb. 2014.

[7] Y. Zeng and R. Zhang, "Optimized training design for wireless energy transfer,' IEEE Trans. Commun., vol. 63, no. 2, pp. 536-550, Feb. 2015.

[8] G. Yang, C. K. Ho, and Y. L. Guan, "Dynamic resource allocation for multiple-antenna wireless power transfer," IEEE Trans. Signal Process., vol. 62, no. 14 , pp. 3565-3577, Jul. 2014.

[9] L. Liu, R. Zhang, and K. Chua, "Multi-antenna wireless powered communication with energy beamforming," IEEE Trans. Commun., vol. 62, no. 12, pp. 4349-4361, Dec. 2014.

[10] J. Xu, L. Liu, and R. Zhang, "Multiuser MISO beamforming for simultaneous wireless information and power transfer," IEEE Trans. Signal Process., vol. 62, no. 18, pp. 4798-4810, Sep. 2014.

[11] S. Kashyap, E. Björnson, and E. G. Larsson, "Can wireless power transfer benefit from large transmitter arrays?" in Proc. IEEE Wireless Power Transfer Conference, 2015.

[12] F. R. Farrokhi, G. J. Foschini, A. Lozano, and R. Valenzuela, "Linkoptimal space-time processing with multiple transmit and receive antennas," IEEE Commun. Lett., vol. 5, no. 3, pp. 85-87, Mar. 2001.

[13] S. M. Kay, Fundamentals of Statistical Signal Processing: Estimation Theory, 1st ed. Prentice Hall Signal Processing Series, 1993, vol. 1.

[14] M. Simon and M.-S. Alouini, Digital Communication over Fading Channels, 2nd ed. Wiley-Interscience, 2005. 